\title{
Similarity and proximity: When does close in space mean close in mind?
}

\author{
Daniel Casasanto \\ Stanford University, Stanford, California
}

\begin{abstract}
People often describe things that are similar as close and things that are dissimilar as far apart. Does the way people talk about similarity reveal something fundamental about the way they conceptualize it? Three experiments tested the relationship between similarity and spatial proximity that is encoded in metaphors in language. Similarity ratings for pairs of words or pictures varied as a function of how far apart the stimuli appeared on the computer screen, but the influence of distance on similarity differed depending on the type of judgments the participants made. Stimuli presented closer together were rated more similar during conceptual judgments of abstract entities or unseen object properties but were rated less similar during perceptual judgments of visual appearance. These contrasting results underscore the importance of testing predictions based on linguistic metaphors experimentally and suggest that our sense of similarity arises from our ability to combine available perceptual information with stored knowledge of experiential regularities.
\end{abstract}

How do people judge the similarity of words, objects, or ideas? Despite concerns about its usefulness as a construct (Goodman, 1972), similarity remains the focus of much psychological research, perhaps because our sense of similarity seems intimately linked with our capacity to generalize, to form categories, and to individuate concepts (Medin, Goldstone, \& Gentner, 1993). This article investigates the relationship between similarity and spatial proximity that is suggested by linguistic metaphors. In English (and many other languages), speakers often talk about similarity by using words and expressions that describe spatial relations. Things that are similar along nearly any dimension can be described as close, and things that are dissimilar as far apart. For example,

1. a. These two shades of blue aren't identical, but they're close.

b. The opposing candidates' stances on the issue couldn't be farther apart.

Does the way people talk about similarity reveal something fundamental about the way they conceptualize it? According to conceptual metaphor theory (Lakoff \& Johnson, 1980, 1999), metaphors in language (e.g., a long time, a deep love, a high price) reveal that many of our abstract concepts depend, in part, on a few simpler concepts grounded directly in perceptuomotor experience. Our notion of similarity is abstract, like our ideas of time, love, or value, insomuch as it is (1) vaguely and variably defined, (2) highly context dependent, and (3) mentalistic, lacking a concrete referent in the physical world that can be perceived through the senses.

Conceptual metaphor theorists posit a functional distinction between concrete source domains such as space, force, and motion, which we can experience through sensory perception and motor action, and more abstract target domains, which we can experience only through interoception or introspection. A cross-domain mapping from a source domain to a target domain can be notated target is source (e.g., similarity is proximity) or alternatively, source $\rightarrow$ target (e.g., proximity $\rightarrow$ similarity) (Lakoff \& Johnson, 1999). In metaphorical language, the target domain is lexicalized by borrowing words or constructions from the source domain. But importantly, by hypothesis, these source-target mappings are not constituted by words; rather, linguistic metaphors are manifestations of nonlinguistic mappings between conceptual or perceptual domains.

The terms metaphor, metaphorical mapping, and conceptual metaphor are often used ambiguously, even by metaphor theorists: Sometimes these terms refer to structures in language, and other times to nonlinguistic mental representations. This ambiguity complicates any discussion of the relationship between metaphorical language and metaphorical thinking. I will distinguish the linguistic and nonlinguistic components of conceptual metaphors by using the terms linguistic metaphor to refer to words and expressions in language and mental metaphor to refer to the associations between nonlinguistic source and target domains that are hypothesized to underlie linguistic metaphors. The notations target is source and source $\rightarrow$ target will refer to mental metaphors.

Despite the prominence of conceptual metaphor and related theories in the cognitive linguistics literature, relatively few behavioral studies have explored the psychological reality of mental metaphors (Murphy, 1996, 1997), and even fewer studies have tested whether people use mental meta-

D. Casasanto, casasanto@alum.mit.edu 
phors when they are not using language (cf. Casasanto \& Boroditsky, 2008). Yet metaphor theory may be of critical importance for advancing embodied theories of mental representation in psychology and cognitive neuroscience (e.g., Barsalou, 1999). Theories of embodied cognition, such as Barsalou's perceptual symbol systems, posit that concepts are constituted, at least in part, by mental simulations of our perceptions and actions. The results of numerous experiments are consistent with this proposal (e.g., Borghi, 2004; Glenberg \& Kaschak, 2002; Tucker \& Ellis, 2004; Zwaan $\&$ Yaxley, 2003), suggesting that mental representations of concrete objects and actions comprise partial recapitulations of perceptuomotor experiences. Abstract concepts, however, present a serious challenge for embodied theories: Thinking about concrete objects and actions may involve perceptuomotor simulations, but how can we perceptually simulate things that we can never perceive? Metaphor theory provides a potential answer: To the extent that perceptuomotor schemas constitute the content of abstract concepts, these concepts can be instantiated by the same neural and mental structures that simulate perception and action in the physical world. Within cognitive linguistics, embodiment theory and metaphor theory appear to be mutually inextricable (e.g., Lakoff \& Johnson, 1999). Proponents of embodied cognition in other areas of cognitive science, however, have marginalized the role of metaphor in the mental representation of abstract concepts (e.g., Barsalou, 1999; Barsalou \& Wiemer-Hastings, 2005; Prinz, 2002). Barsalou, for example, argues that "a direct, non-metaphorical representation of an abstract domain is essential" and proposes that "perceptual symbol systems can represent all abstract concepts directly" (p. 600). This stance is somewhat surprising, since mental metaphors seem a likely component of the solution to the problem of representing the imperceptible via perceptuomotor simulations. A goal of the present article is to provide two necessary precursors to the incorporation of metaphor into psychological theories of embodied cognition: an explicit proposal for how (at least some) mental metaphors are created and a method for testing the activation of a particular mental metaphor that uses both linguistic and nonlinguistic stimuli and responses.

Mental metaphors linking concrete and abstract domains may become established as we implicitly track useful correlations in our physical experience (Casasanto, in press; Lakoff \& Johnson, 1980, 1999). For example, the mental metaphor proximity $\rightarrow$ similarity could plausibly arise as we implicitly learn that, in both the natural and the human-made environment, more similar things tend to be found closer together in space, and less similar things farther apart (imagine the clustering of wildflowers in a field, the categorization of books in a library, or the arrangement of products on supermarket shelves). This pattern in which similar things tend to clump together in space was noted by the Gestalt psychologists (e.g., Wertheimer, 1923/1938) and by linguists interested in relationships among language, concepts, and space (Jackendoff, 1983; Lakoff \& Johnson, 1999). Over time, as we perceive and act upon our "clumpy" environment, we habitually coactivate information about space and similarity, giving rise to a functional connection between these mental domains. Information in one domain can then be used heuristically as an index of the other. In particular, information in the more concrete, perceptually available domain can be used to support inferences in the more abstract domain (e.g., spatial information is used heuristically to support inferences about similarity). A directed connection between habitually coactivated mental representations of proximity and similarity could be implemented at a neural level by a process such as Hebbian learning (Hebb, 1949). On this view, similarity relationships are not merely analogous to spatial relationships. Rather, once a cross-domain mapping has been established, spatiomotor representations that can be used for reasoning about physical spatial relationships are also activated automatically to support reasoning in abstract, nonspatial domains such as similarity (Casasanto, in press; Casasanto \& Boroditsky, 2008; Gallese \& Lakoff, 2005; Lakoff \& Johnson, 1999). ${ }^{1}$

The mental metaphor proximity $\rightarrow$ similarity figures prominently in Lakoff and Johnson's (1999) exposition of conceptual metaphor theory. They choose this example to illustrate the relationship between source and target domains (p. 58) and to argue that without such crossdomain mappings, abstract thought is "virtually impossible" (p. 59). There is compelling evidence that people talk about similarity using spatial words, but do people use the corresponding spatial schemas to reason about similarity even when they are not producing or understanding language? This question cannot in principle be addressed on the basis of linguistic or psycholinguistic data alone.

The experiments reported here tested the hypothesis that our notion of similarity depends in part on mental representations of physical distance, as predicted by spatial metaphors for similarity in language and by the proposed mental metaphor proximity $\rightarrow$ similarity. Lakoff and Johnson (1999, p. 59) analyzed this mental metaphor into two complementary mappings: spatial closeness $\rightarrow$ similarity, and spatial distance $\rightarrow$ dissimilarity, which are predicted on the basis of expressions in language such as 1a and $1 \mathrm{~b}$ above. The present experiments tested whether these crossdomain mappings are also evident in explicit similarity judgments about both linguistic and nonlinguistic stimuli. In three experiments, participants rated the similarity of pairs of words or pictures, which were presented at varying distances on the computer screen (e.g., close, medium, or far apart). Across participants, a given stimulus pair appeared an equal number of times at each spatial distance. If the spatial relationships encoded in linguistic metaphors are automatically activated during the process of evaluating similarity - whether or not evaluations involve linguistic stimuli or responses - the participants should judge stimuli to be more similar when they are presented closer together on the screen than when they are presented farther apart.

\section{EXPERIMENT 1 Abstract Nouns}

Experiment 1 tested whether participants would rate pairs of abstract nouns to be more similar in meaning when they appeared closer together on the screen than when they ap- 
peared presented farther apart. Abstract nouns (e.g., grief, justice, hope) were chosen as stimuli for this first test of the relationship between similarity and proximity because their meanings are notoriously vague and context dependent; therefore, judgments of their similarity in meaning may be particularly susceptible to the influence of task-irrelevant variations in the spatial distance between stimuli.

\section{Method}

Participants. Twenty-seven native English speakers from the Stanford University community participated in exchange for payment.

Materials. Seventy-two abstract nouns (concreteness rating $<400$ ) between 4 and 10 letters long were selected from the MRC Psycholinguistic Database. The nouns were randomly combined into 36 pairs (e.g., grief-justice, memory-hope, sympathyloyalty; see Appendix A). Words were presented on an iMac monitor $(1,024 \times 768$ pixel resolution $)$ in 14-point Courier font.

Design and Procedure. The participants viewed all 36 word pairs in randomized order and rated their similarity in meaning (i.e., "How similar in meaning?") on a scale from 1 (not at all similar) to 9 (very similar). Each word appeared centered within one of two "picture frames" (150 pixels wide, 50 pixels high) on the vertical midline of the screen. The centers of the frames were separated horizontally by
150 pixels in the close condition, 300 pixels in the medium condition, and 450 pixels in the far condition. Distance between the stimuli was manipulated within subjects: For each participant, one third of the word pairs appeared at each of the three distances. The participants saw each word pair only once, and the assignment of word pairs to distance conditions was counterbalanced across participants.

In order to reduce demand characteristics of the task, the close, medium, and far picture frames appeared in one of four different positions on the far left, middle left, middle right, or far right of the screen. Within each distance condition (i.e., close, medium, and far), an equal number of stimulus pairs was randomly assigned to each of the left-right positions. As a result, there was a total of 12 different configurations of picture frames. The midpoint between the picture frames was on the left side of the screen for half of the trials and on the right side for the other half. Variation in the left-right position of the stimuli was orthogonal to the variation in distance between them and, thus, provided a highly salient filler variable.

At the beginning of each trial, two empty picture frames appeared simultaneously and remained visible until the end of the trial. After $500 \mathrm{msec}$, the first word of each pair appeared in the left picture frame for 2,000 msec and then disappeared. After a 500-msec interstimulus interval, the second word of the pair appeared in the right picture frame for 2,000 $\mathrm{msec}^{2}$ As soon as the second word disappeared, a response prompt and the 9-point scale appeared, and

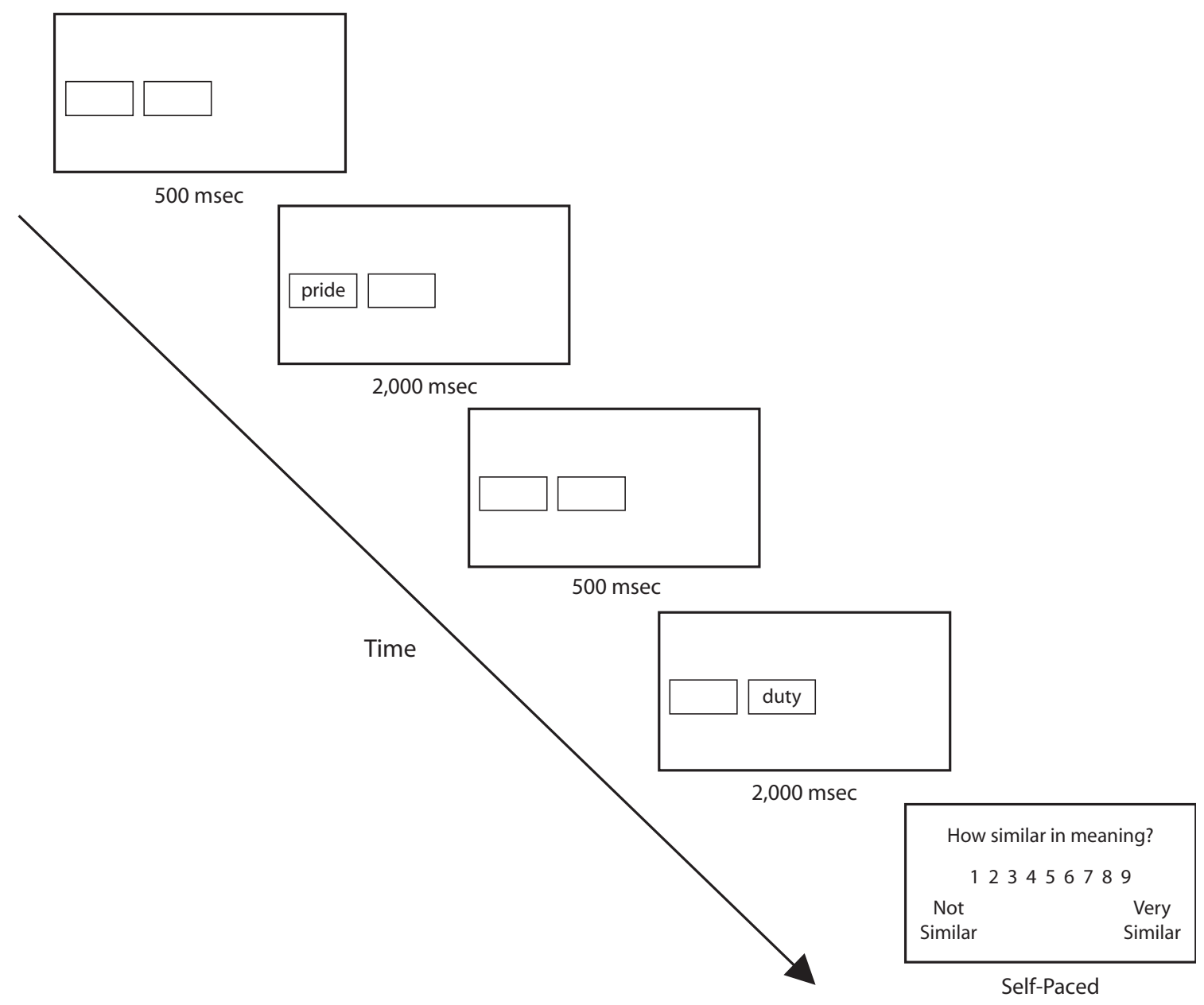

Figure 1. Schematic representation of the trial structure for Experiment 1 (close condition). 
the participants responded by pressing a number key on a standard keyboard (see Figure 1). Responses were self-paced.

\section{Results and Discussion}

The results of Experiment 1 showed that stimuli were judged to be more similar when they were presented closer together than when they were farther apart. Average similarity ratings were $3.80(S E=0.19)$ in the close condition, $3.33(S E=0.12)$ in the medium condition, and $3.28(S E=0.15)$ in the far condition. Ratings were normalized by participants (for analysis by participants) and by items (for analysis by items), and Z-scored similarity ratings were compared using a one-way ANOVA (see Figure 2). ${ }^{3}$ Ratings differed significantly across conditions, both by participants $\left[F_{1}(2,52)=3.45, p=.04\right]$ and by items $\left[F_{2}(2,105)=4.49, p=.02\right]$.

One possibility is that the influence of spatial proximity may have been restricted to low-similarity items, for which word meanings were difficult to compare. To address this concern, an analysis was conducted to determine whether the relationship between proximity and similarity differed for items that were judged to have high similarity (e.g., sympathy-love) versus low similarity (e.g., deductionglory). Items were mean-split according to their overall similarity ratings. A two-way ANOVA showed no significant interaction of similarity (high or low) and distance (close, medium, or far) $[F(2,102)=1.90$, n.s.]; thus, the effect of distance on similarity ratings did not differ between high- and low-similarity word pairs.

The finding that stimuli were rated more similar when presented closer together is consistent with predictions based on conceptual metaphor theory. A further concern in interpreting these results was that perhaps they were consistent with predictions based on linguistic metaphors only because the stimuli were (1) linguistic and (2) highly abstract. Would the same pattern of results be found for similarity judgments on nonlinguistic stimuli that have more concrete, perceptible features? This question was addressed in Experiments 2 and 3.

\section{EXPERIMENT 2 Unfamiliar Faces}

Experiment 2 tested whether the results of Experiment 1 would generalize to a different type of stimulus, for which similarity had to be computed along different dimensions. To judge the similarity of abstract noun pairs, participants had to retrieve word meanings from memory and to reason about unseen properties of abstract entities. Because the appearance of words is arbitrarily related to their meaning, the visual stimuli themselves provided little information (if any) that was relevant to the similarity judgment. Would distance still influence similarity judgments, as in Experiment 1, even if more of the relevant information were given perceptually in the visual stimuli themselves? According to conceptual metaphor theory, it should.

People use spatial metaphors in language to describe similarities between abstract and concrete things alike. Just as two abstract words can be said to be close in meaning, two paint chips can be close in color, two lines can be close

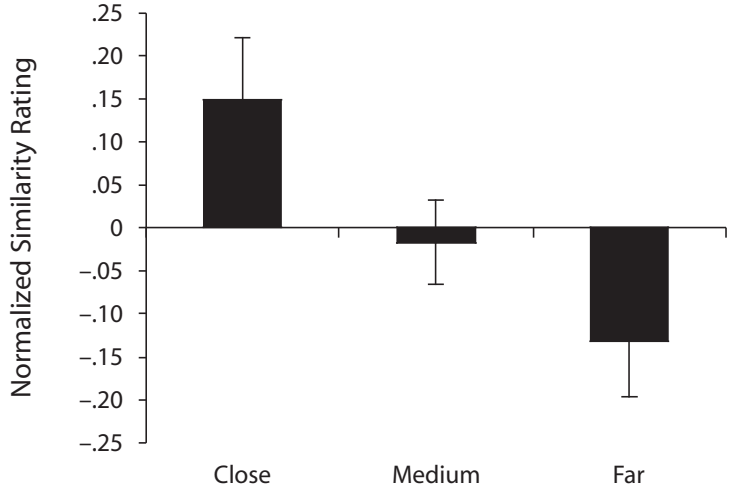

Figure 2. Results of Experiment 1. Similarity ratings for pairs of abstract nouns varied significantly as a function of their spatial separation on the screen, which was consistent with predictions based on spatial metaphors for similarity in language. Error bars indicate standard errors of the means.

in length, two shirts can be close in size, and two faces can be close in appearance. The relationship between similarity and proximity in linguistic metaphors generalizes broadly (so broadly, in fact, that it is difficult to imagine a case in which similarity cannot be described in terms of closeness). The same linguistic metaphor can describe similarity along both conceptual and perceptual dimensions. Therefore, if people conceptualize similarity the way they talk about it, the same prediction about the relationship between similarity and proximity should hold for both conceptual judgments about abstract entities and perceptual judgments about more concrete entities.

For Experiment 2, the participants judged the similarity of pairs of unfamiliar faces. Whereas the participants in Experiment 1 were instructed to judge similarity of abstract words on the basis of their meanings, the participants in Experiment 2 were instructed to judge similarity of faces based on their visual appearance (i.e., "How similar in appearance?"), on a scale from 1 (not at all similar) to 9 (very similar).

\section{Method}

Participants. Thirty-three native English speakers from the MIT community participated in exchange for payment.

Materials and Procedure. Sixty pairs of unfamiliar faces were constructed from a database of University of Pennsylvania ID card photos. Half were male-male pairs, and half were female-female pairs. The face pairs were presented exactly as the word pairs had been presented in Experiment 1, with the following exception: The height of the "picture frames" was changed to accommodate the size of the photos ( 150 pixels wide $\times 200$ pixels high).

\section{Results and Discussion}

The results of Experiment 2 showed that stimuli were judged to be more similar when they were presented farther apart than when they were presented closer together, contrary to predictions based on spatial metaphors for similarity in language. Average similarity ratings were $3.87(S E=0.06)$ in the close condition, $4.01(S E=0.07)$ in the medium condition, and $4.18(S E=0.07)$ in the 


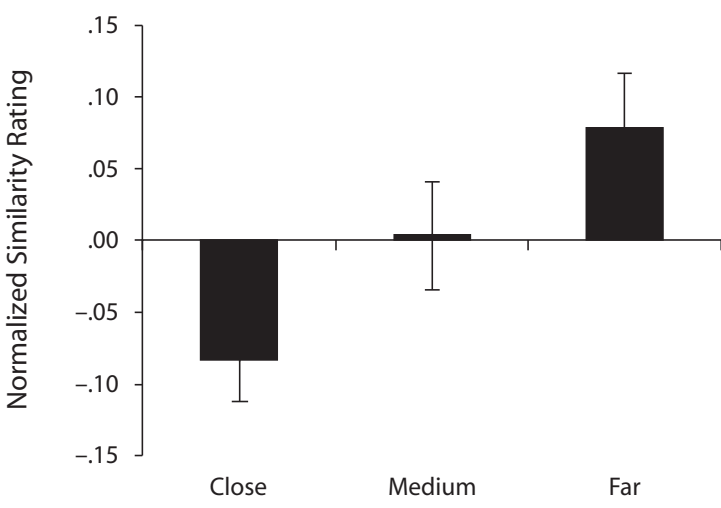

Figure 3. Results of Experiment 2. Similarity ratings for pairs of faces varied significantly as a function of their spatial separation on the screen, but contrary to predictions based on spatial metaphors for similarity in language. Error bars indicate standard errors of the means.

far condition. Ratings were normalized by participants (for analysis by participants) and by items (for analysis by items), and $Z$-scored similarity ratings were compared using a one-way ANOVA (Figure 3). Ratings differed significantly across conditions (although not in the predicted direction), both by participants $\left[F_{1}(2,64)=3.61, p=.04\right]$ and by items $\left[F_{2}(2,177)=3.29, p=.04\right]$.

As for Experiment 1, an analysis was conducted to determine whether the relationship between proximity and similarity differed overall for items that were judged to have high similarity versus low similarity. Items were mean-split according to their overall similarity ratings. A two-way ANOVA showed no significant interaction of similarity (high or low) and distance (close, medium, or far) $[F(2,174)=1.18$, n.s. $]$; thus, the effect of distance on similarity ratings did not differ between high- and lowsimilarity face pairs.

\section{EXPERIMENT 3 Object Pictures}

Why did proximity have opposite effects on similarity ratings for abstract nouns and unfamiliar faces? Experiments 1 and 2 differed both in the kind of stimulus the participants judged (i.e., verbal vs. pictorial) and in the kind of judgments they made (i.e., conceptual judgments based on meaning vs. perceptual judgments based on visual appearance). Experiment 3 evaluated whether the results of Experiments 1 and 2 differed because of the type of stimulus or the type of judgment.

For Experiment 3, different judgments were made on the same set of stimulus pictures, which depicted common objects. Half of the participants were instructed to judge their similarity in visual appearance (a perceptual judgment), and the other half to judge their similarity in function or use (a conceptual judgment). If the contrast between the results of Experiments 1 and 2 was due to a difference in the type of experimental materials used, the results of both the perceptual and the conceptual judgments in Experiment 3 should resemble those in Experiment 2 , in which pictorial stimuli were used: Closer stimuli should be judged to be less similar, regardless of the type of judgment the participants made. This outcome would yield a main effect of distance (close or far) but no interaction of distance and judgment type (perceptual or conceptual). Alternatively, if the difference between results of the first two experiments was due to the participants' judging abstract, unseen properties of the stimuli in Experiment 1 but judging concrete, perceptible properties of the stimuli in Experiment 2, then in the present experiment, the results of conceptual judgments should be similar to those in Experiment 1 (i.e., closer stimuli should be judged more similar), whereas the results of perceptual judgments should be similar to those in Experiment 2 (i.e., closer stimuli should be judged less similar). This outcome would yield an interaction of distance (close or far) and judgment type (perceptual or conceptual).

\section{Method}

Participants. Eighty native English speakers from the MIT community participated in Experiment 3 in exchange for payment. Forty participants were randomly assigned to the conceptual judgment condition, and the other 40 to the perceptual judgment condition.

Materials and Procedure. Thirty pairs of objects were constructed from the Snodgrass and Vanderwart (1980) line drawings. Objects were paired only within semantic categories (e.g., tools, clothing, furniture) to facilitate meaningful judgments of functional similarity in the conceptual judgment condition (see Appendix B). The participants viewed all 30 object pairs in randomized order. The participants assigned to the conceptual judgment condition rated their functional similarity (i.e., "How similar in function or use?") on a scale from 1 (not at all similar) to 9 (very similar), whereas the participants assigned to the perceptual judgment condition rated their visual similarity (i.e., "How similar in visual appearance?") on the same scale. Object pairs appeared in "picture frames" (150 pixels wide $\times 200$ pixels high) and were presented exactly as in the previous experiment, with the following exceptions: The stimuli appeared at one of two distances on the screen (instead of three), without the orthogonal left-right variation in position used previously, in order to maximize the difference between the close condition (in which the centers of pictures were separated by 150 pixels) and the far condition (in which the centers of pictures were separated by 600 pixels). Distance between stimuli was manipulated within subjects, and the assignment of picture pairs to distance conditions was counterbalanced across participants.

\section{Results and Discussion}

The results showed that during conceptual judgments, closer stimuli were judged to be more similar. Average similarity ratings were $6.15(S E=0.04)$ in the close condition and $5.97(S E=0.04)$ in the far condition. By contrast, during perceptual judgments, closer stimuli were judged to be less similar. Average similarity ratings were 5.12 $(S E=0.04)$ in the close condition and $5.28(S E=0.04)$ in the far condition. Similarity ratings were normalized by participants (for analysis by participants) and by items (for analysis by items), and $Z$-scored ratings were compared using a two-way ANOVA (see Figure 4). A mixed ANOVA with distance (close or far) as a within-subjects factor and judgment type (perceptual or conceptual) as a between-subjects factor showed a significant interaction by participants $\left[F_{1}(1,78)=12.23, p=.001\right]$. This signifi- 


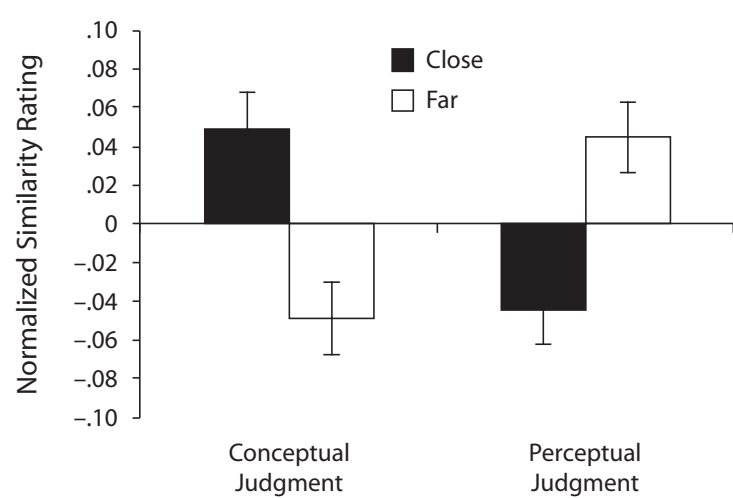

Figure 4. Results of Experiment 3. Similarity ratings for pairs of object pictures varied significantly as a function of their spatial separation on the screen. The relationship between proximity and similarity was consistent with predictions based on spatial metaphors for similarity in language during conceptual judgments (left) but was inconsistent during perceptual judgments of the same stimuli (right). Error bars indicate standard errors of the means.

cant interaction was confirmed in a two-way ANOVA by items $\left[F_{2}(1,116)=12.12, p=.001\right]$.

An additional analysis was performed to compare the effect of distance on similarity ratings for close versus far trials across Experiments 1, 2, and 3. The effect of proximity on similarity judgments for each experiment was defined as the difference between participants' mean similarity ratings in the close and far conditions [effect of proximity on similarity $=$ (mean of normalized similarity ratings in the close condition) - (mean of normalized similarity ratings in the far condition)] and was compared across all the experiments, using a one-way ANOVA $[F(3,136)=8.81, p=.0001$; see Figure 5]. Two-tailed pairwise independent-samples $t$ tests showed significant differences between the effects of proximity on similarity ratings for abstract nouns versus perceptual object judgments [difference $=.37 ; t(56)=3.28, p=.002$ ], abstract

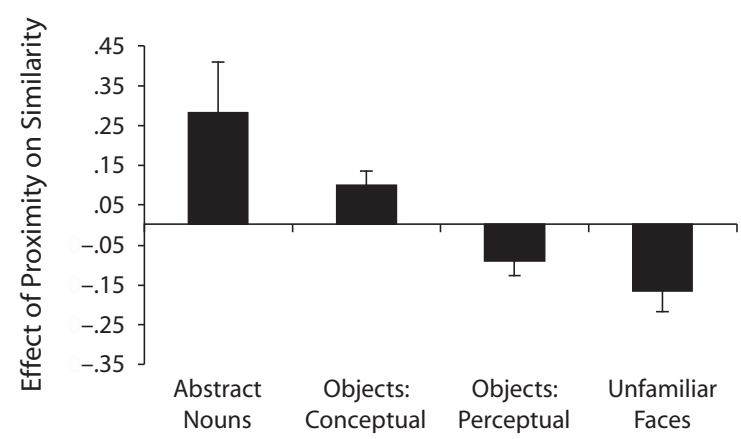

Figure 5. Comparison of the effect of proximity on similarity ratings across experiments. Closer stimuli were rated more similar during conceptual judgments (left columns: Experiments 1 and 3 , conceptual judgment condition) but less similar during perceptual judgments (right columns: Experiment 3, perceptual judgment condition, and Experiment 2). Error bars indicate standard errors of the means. nouns versus faces [difference $=.44 ; t(58)=3.41, p=$ $.001]$, perceptual object judgments versus conceptual object judgments [difference $=.19 ; t(78)=3.57, p=$ $.001]$, and conceptual object judgments versus faces [difference $=.26 ; t(71)=3.98, p=.0001]$. Importantly, no differences were found between the effects of proximity on similarity ratings for abstract nouns versus conceptual object judgments [difference $=.18 ; t(65)=1.62$, n.s.] or for perceptual object judgments versus faces [difference $=.07 ; t(71)=1.14$, n.s.]. In summary, this analysis shows that all pairwise comparisons between judgment types (conceptual vs. perceptual) yielded highly significant differences, whereas pairwise comparisons within judgment types yielded no significant differences.

Together, the results of Experiment 3 and of the comparative analysis indicate that the contrasting effects of proximity on similarity judgments found in Experiments 1 and 2 were not due to superficial differences between the verbal and the pictorial stimuli. Rather, the effect of proximity on similarity depends on the kind of judgment participants make: conceptual judgments about abstract entities or unseen object properties versus perceptual judgments about visible stimulus properties.

In addition, this overall pattern of results helps to address any residual concerns regarding the demand characteristics of the tasks. It is plausible, a priori, that the participants may have become aware of the proximity manipulation and focused their attention on it despite the highly salient variation in the left-right position of the stimuli, which was orthogonal to the variation in their spatial separation (see the Method section in Experiment 1). If the participants noticed that some stimuli appeared closer together than others, they could have consciously accessed the association between similarity and proximity found in linguistic metaphors. This could potentially account for the results for the conceptual similarity judgments, which were consistent with spatial metaphors for similarity in language, but this explanation cannot account for the results for the perceptual judgments, in which the effect of distance on similarity judgments ran contrary to linguistic metaphors. Furthermore, in order to explain the interaction of distance and judgment type in Experiment 3 in terms of demand characteristics, it would be necessary to explain why the participants imputed different experimental demands during conceptual judgments versus perceptual judgments on the same stimuli.

\section{GENERAL DISCUSSION}

Experiments 1-3 tested whether similarity ratings for words and pictures vary as a function of how far apart stimuli appear on a computer screen. The results showed that spatial proximity influenced similarity judgments significantly in all the experiments, but the direction of influence varied according to the type of judgment the participants made. Closer stimuli were rated more similar during conceptual judgments of abstract entities or unseen object properties but less similar during perceptual judgments of the visual appearance of faces and objects. Conceptual judgments followed predictions based on linguistic meta- 
phors that express similarity in terms of spatial proximity: When stimuli appeared closer in physical space, they were judged to be "closer" in participants' mental similarity space, as well. Perceptual judgments showed the opposite pattern, however, contrary to predictions based on linguistic metaphors for similarity. Overall, the results suggest that our notion of similarity depends, in part, on our experience of spatial distance, yet they also show that we cannot necessarily infer the relationship between similarity and proximity in people's nonlinguistic mental representations from patterns in metaphorical language alone.

Conceptual metaphor theory (Lakoff \& Johnson, 1980, 1999) cannot easily account for the difference between the effects of distance on perceptual versus conceptual judgments, given that the same spatial metaphors can be used to describe similarities in both low-level perceptual properties and high-level conceptual properties: Similarities in appearance, function, or meaning can all be described using words like close and far. Linguistic metaphors suggest that the same mental metaphor, proximity $\rightarrow$ similarity, underlies our notions of both perceptual and conceptual similarity. Thus, language predicts that distance should have the same effect on perceptual and conceptual similarity judgments, contrary to the results of Experiments 1-3.

Is it possible that conceptual metaphor can accommodate these data on a looser, higher level interpretation of the theory? The fact that every experiment showed significant effects of spatial distance on similarity judgments validates an important link between the similarity and space, so perhaps these results should be considered consistent with a broader construal of metaphor theory. Perhaps, but this interpretation is problematic. Conceptual metaphor does not predict just any relationship between similarity and space; it predicts two specific complementary relationships: spatial closeness $\rightarrow$ similarity and spatial distance $\rightarrow$ dissimilarity (Lakoff \& Johnson, 1999, p. 59). Spatial closeness $\rightarrow$ dissimilarity is not hypothesized to be a mental metaphor, and there is no corresponding linguistic metaphor: To say that two shades of blue are close when you mean that they are dissimilar would be either ironic or nonsensical.

If the present results are interpreted as validating conceptual metaphor theory overall, then the theory is rendered too vague to make falsifiable behavioral predictions. On a more fruitful interpretation, metaphors in language remain a powerful predictor of relationships between nonlinguistic domains of knowledge in general; and behavioral results, such as those presented here, that systematically confirm or disconfirm predictions about conceptual structure that are based on linguistic metaphors will eventually help to clarify metaphor theory and establish its boundaries. Although some null results have been reported previously (e.g., Bergen, Lindsay, Matlock, \& Narayanan, 2007), data from Experiments 2 and 3 (perceptual judgment condition) may be the first experimental results to demonstrate significant effects that directly contradict predictions that follow from conceptual metaphor theory.

Like the results of Experiments 1 and 3 (conceptual judgment condition), previous studies have also shown positive associations between proximity and conceptual similarity. Sweetser (1997) observed that speakers some- times bring their hands closer together in space to indicate the similarity of abstract ideas during spontaneous cospeech gestures. Goldstone (1994) asked participants to arrange various tokens of the letter " $\mathrm{A}$ " on the computer screen so that more similar tokens were positioned closer in space. Importantly, although, in principle, similarity between tokens of the letter " $A$ " could depend on the perceptual properties of the stimuli, Goldstone noted that when the participants were asked to indicate similarity via spatial proximity, they focused on "abstract commonalities" between tokens (p. 385). Whereas the participants' evaluations of the "A" stimuli were driven by perceptual similarity for nonspatial judgments (i.e., same/different judgments), instructing the participants to arrange stimuli according to the rule that closer $=$ more similar led them to "tap into a level of similarity that is relatively cognitive rather than perceptual" (p. 385). This complex relationship between spatial proximity, conceptual similarity, and perceptual similarity appears to have been unexpected in the Goldstone study, as it was in the present study.

It may be possible to account for the results of Experiments $1-3$ by positing that physical closeness influences our assessment of similarity in two contrasting ways $(2 \mathrm{a}-2 \mathrm{~b})$ :

2. a. Physical closeness encourages noticing perceptible differences between stimuli.

b. Physical closeness encourages construing stimuli as members of the same category.

Figure 6 illustrates that moving items closer together in space, even by a few centimeters, can dramatically increase the salience of simple perceptible differences that are hard to notice when the same items are presented farther apart. ${ }^{4}$ In this way, proximity can make closer stimuli seem less similar. For more complex stimuli, proximity may encourage zooming in attention on fine-grained perceptual details that might not appear relevant when the same items are presented farther apart. These effects of proximity on similarity should be particularly relevant for making perceptual judgments (as in Experiments 2 and 3, perceptual condition) but less relevant for making conceptual judgments (as in Experiments 1 and 3, conceptual condition).

By contrast, proximity also encourages construing stimuli as members of the same category, and categorizing things together makes them seem more similar (Goldstone, Lippa, \& Shiffrin, 2001). Relationships among similarity, proximity, and category membership that are found in our environment may help to explain why construing

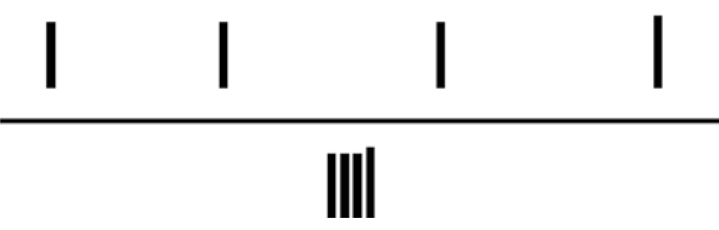

Figure 6. The vertical bars above the horizontal line are identical in length to the bars below the horizontal line. This figure demonstrates that proximity can facilitate noticing small differences along perceptual dimensions (e.g., height). 
things as members of the same category increases their similarity. As Gestalt psychologists have noted, the environment is pervasively clumpy: At various scales and levels of abstraction, things that belong to the same category tend to be found close together and also tend to be more similar to one another than do things that belong to different categories (Wertheimer, 1923/1938). We are continually exposed to the clumping together of things that are similar, both in the organization of the natural world and in our human-made surroundings. Imagine picking one flower in a field of various wildflowers and then picking another flower that is either right next to it or one that is 10 paces away. The closer flower is more likely to belong to the same species as the first (i.e., the same category of flowers) and, therefore, to be more similar to the first than is the flower that is farther away. Where clumping does not occur naturally, people often create it: Consider the organization of products on supermarket shelves or books in a library; clumping can be observed from the small-scale arrangement of utensils in the silverware drawer to the large-scale distribution of wealth, race, or religion across neighborhoods or nations. Implicitly tracking this clumpiness may be useful for reasoning about our environment in ways that predict contingencies and guide behavior (Anderson, 1991; Shepard, 1987; Tenenbaum \& Griffiths, 2001). Building on Wertheimer's principles of proximity and similarity, it is plausible that people implicitly learn and use a set of relationships that could be called the clumpiness principle (3):

\section{3 . The clumpiness principle: proximity $\alpha$ similarity $\alpha$ category membership.}

If people use stored knowledge of clumpiness heuristically when judging similarity, they should rely on this heuristic more when relevant perceptible data are scarce than when they are abundant (in which case, such heuristics can be ignored in favor of the perceptible information at hand). As such, the influence of proximity on similarity that follows from the heuristic use of the clumpiness principle should be particularly relevant for making conceptual judgments (as in Experiments 1 and 3, conceptual conditions) but less relevant for making perceptual judgments (as in Experiments 2 and 3, perceptual conditions).

On this proposal, proximity exerted two contrasting influences on similarity judgments in Experiments 1-3. When perceptible information was available in the stimuli (and was relevant to the task), the participants used it, judging closer stimuli to be less similar in Experiments 2 and 3 (perceptual judgment conditions) because proximity encouraged noticing small perceptible differences that were less noticeable when the stimuli were presented farther apart. By contrast, when perceptual information was not available in the stimuli (in Experiment 1) or was not relevant to the required judgment (in Experiment 3, conceptual judgment condition), closer stimuli were judged to be more similar, reflecting the participants' heuristic use of the knowledge that, in our clumpy environment, proximity correlates with similarity (perhaps mediated by the implicit knowledge that proximity also correlates with category membership).
It may be possible to integrate these contrasting influences of spatial proximity on conceptual and perceptual similarity judgments if the computation of similarity is considered to be a process of rational inference that optimally combines perceptible information at hand with stored knowledge of experiential regularities in the environment (Anderson, 1991; Shepard, 1987; Tenenbaum \& Griffiths, 2001). Tenenbaum and Griffiths proposed a Bayesian model, according to which the similarity between two items is computed in terms of the probability that they are members of the same category (i.e., drawn from the same statistical distribution). In their model, the probability that items share category membership is proportional to the likelihood that they do so given the information present in the stimuli per se and to the probability that they do so given the observer's prior experience and stored knowledge. When the participants in the present experiments performed conceptual similarity judgments, there was little information in the stimuli per se on which they could base their judgments, so their estimates of the probability that stimulus items belonged to the same category (and were therefore similar) were likely to depend strongly on their prior knowledge, which includes implicit knowledge of the clumpiness principle. By contrast, when the participants were performing perceptual similarity judgments, their estimates of the probability that stimulus items belonged to the same category were likely to depend more strongly on the information given in the stimuli themselves, which overwhelmed any influence of the clumpiness principle.

Although the difference between the effects of spatial proximity on conceptual and perceptual similarity judgments found in Experiments 1 and 2 was not predicted a priori, this difference was replicated in the conceptual and perceptual judgment conditions in Experiment 3. The comparative analysis across experiments confirmed that what determined whether proximity made stimuli seem more similar or less similar was the type of judgment that the participants made (conceptual or perceptual), not the type of items that they judged (verbal or pictorial). A question for further research is whether the pattern of results discovered here might generalize beyond the laboratory. Do we think that two politicians are more similar in appearance when we see them sitting farther apart, but find their views on the issues to be more alike when we see them sitting closer together? These experiments raise the possibility that spatial proximity may subtly influence the similarity judgments we make, implicitly or explicitly, in everyday settings.

\section{CONCLUSIONS}

Three experiments demonstrated that similarity ratings for pairs of words and pictures were influenced systematically by their spatial separation on the computer screen. In all the experiments, similarity judgments were affected by task-irrelevant variations in spatial proximity, but not always as predicted by spatial metaphors in language. When the participants made conceptual judgments about abstract entities or unseen object properties, stimuli presented closer 
together were judged to be more similar than stimuli presented farther apart, consistent with predictions based on linguistic metaphors linking similarity to physical closeness (Lakoff \& Johnson, 1999). By contrast, when the participants made perceptual judgments about visible stimulus properties, stimuli presented closer together were judged to be less similar than stimuli presented farther apart, contrary to predictions based on linguistic metaphors.

These findings underscore the importance of testing conceptual metaphor theory experimentally, and suggest that it is not possible to infer the relationship between similarity and proximity in people's nonlinguistic mental representations solely on the basis of patterns in metaphorical language. Even when linguistic metaphors failed to predict the exact relationships revealed by behavioral tests, however, they still pointed to an underexplored link between physical space and our abstract notion of similarity. Proximity and similarity are not unrelated; rather, they appear to be related in more complex ways than linguistic analyses alone can discover. As such, linguistic metaphors should be treated as a source of hypotheses about the structure of abstract concepts. These experiments encourage further exploration of relationships between similarity and proximity and of how people integrate available perceptual information with their implicit knowledge of experiential regularities when making similarity judgments.

\section{AUTHOR NOTE}

This research was supported in part by an NSF Graduate Research Fellowship, NRSA Postdoctoral Fellowship F32MH072502, and Grant SEJ2006-04732/PSIC, DGI from the Spanish Ministry of Education and Science to D.C. Correspondence concerning this article should be addressed to D. Casasanto, Department of Psychology, Stanford University, Jordan Hall, Bldg. 420, Stanford, CA 94305 (e-mail: casasanto@alum mit.edu).

\section{REFERENCES}

Anderson, J. R. (1991). The adaptive nature of human categorization. Psychological Review, 98, 409-429.

Barsalou, L. W. (1999). Perceptual symbol systems. Behavioral \& Brain Sciences, 22, 577-609.

Barsalou, L. W., \& Wiemer-Hastings, K. (2005). Situating abstract concepts. In D. Pecher \& R. Zwaan (Eds.), Grounding cognition (pp. 129-163). Cambridge: Cambridge University Press.

Bergen, B. K., Lindsay, S., Matlock, T., \& Narayanan, S. (2007). Spatial and linguistic aspects of visual imagery in sentence comprehension. Cognitive Science, 31, 733-764.

BorghI, A. M. (2004). Object concepts and action: Extracting affordances from objects parts. Acta Psychologica, 115, 69-96.

Casasanto, D. (in press). Who's afraid of the big bad Whorf? Crosslinguistic differences in temporal language and thought. Language Learning.

Casasanto, D., \& Boroditsky, L. (2008). Time in the mind: Using space to think about time. Cognition, 106, 579-593.

Gallese, V., \& LAKoff, G. (2005). The brain's concepts: The role of the sensory-motor system in conceptual knowledge. Cognitive Neuropsychology, 22, 455-479.

Glenberg, A. M., \& KaschaK, M. P. (2002). Grounding language in action. Psychonomic Bulletin \& Review, 9, 558-565.

Goldstone, R. [L.] (1994). An efficient method for obtaining similarity data. Behavior Research Methods, Instruments, \& Computers, 26, 381-386.

Goldstone, R. L., LiPPA, Y., \& ShifFrin, R. M. (2001). Altering object representations through category learning. Cognition, 78, 27-43.
Goodman, N. (1972). Seven strictures on similarity. In N. Goodman (Ed.), Problems and projects (pp. 437-447). New York: BobbsMerrill.

Heвв, D. O. (1949). The organization of behavior. New York: Wiley.

JACKENDOFF, R. (1983). Semantics and cognition. Cambridge, MA: MIT Press.

LAKOFF, G., \& Johnson, M. (1980). The metaphorical structure of the human conceptual system. Cognitive Science, 4, 195-208.

LAKoff, G., \& JoHnson, M. (1999). Philosophy in the flesh: The embodied mind and its challenge to Western thought. Chicago: University of Chicago Press.

Medin, D. L., Goldstone, R. L., \& Gentner, D. (1993). Respects for similarity. Psychological Review, 100, 254-278.

Murphy, G. L. (1996). On metaphoric representation. Cognition, 60, 173-204.

Murphy, G. L. (1997). Reasons to doubt the present evidence for metaphoric representation. Cognition, 62, 99-108.

Prinz, J. J. (2002). Furnishing the mind: Concepts and their perceptual basis. Cambridge, MA: MIT Press.

Richardson, D. C., \& Spivey, M. J. (2000). Representation, space and Hollywood Squares: Looking at things that aren't there anymore. Cognition, 76, 269-295.

ShePARD, R. N. (1987). Toward a universal law of generalization for psychological science. Science, 237, 1317-1323.

SNODGRASs, J. G., \& VANDERWART, M. (1980). A standardized set of 260 pictures: Norms for name agreement, image agreement, familiarity, and visual complexity. Journal of Experimental Psychology: Human Learning \& Memory, 6, 174-215.

SWEETSER, E. E. (1997, July). Regular metaphoricity in gesture: Bodilybased models of speech interaction. Paper presented at the 16e Congrès International des Linguistes, Paris.

Tenenbaum, J. B., \& Griffiths, T. L. (2001). Generalization, similarity, and Bayesian inference. Behavioral \& Brain Sciences, 24, 629-640.

Tucker, M., \& Ellis, M. (2004). Action priming by briefly presented objects. Acta Psychologica, 116, 185-203.

Wertheimer, M. (1938). Laws of organization in perceptual forms. In W. D. Ellis (Ed.), A source book of Gestalt psychology (pp. 71-88). New York: Harcourt, Brace. (Original work published 1923)

ZwaAn, R. A., \& YAXLEY, R. A. H. (2003). Spatial iconicity affects semantic relatedness judgments. Psychonomic Bulletin \& Review, 10, 954-958.

\section{NOTES}

1. This proposal presupposes that primitive representations in abstract target domains exist prior to the creation (or elaboration) of cross-domain mappings from concrete source domains.

2 . The words of each pair were presented serially, rather than simultaneously, in order to standardize the amount of time that the participants spent on each member of the stimulus pair and also to give the participants ample time (i.e., $500 \mathrm{msec}$ ) to move their eyes from the location of the first stimulus to the location of the second, so that they could foveate each stimulus in turn. In a pilot version of Experiment 1, abstract nouns were presented simultaneously, rather than serially, and remained on the screen until the participant responded. Pilot results did not differ significantly from the results reported here, which are summarized in Figure 2. However, it was not clear from the pilot results what accounted for the difference in similarity judgments. In order to constrain the interpretation of the results reported in this article, the stimuli were presented serially. This rules out low-level explanations for observed differences in similarity ratings, suggesting that they are not due to differences in saccadic activity, peripheral versus foveal viewing of stimuli, or sharing of visual attention across the three distance conditions.

3. The results of Experiments 1-3 are presented in normalized form to facilitate comparison of effects across experiments.

4. Since all the stimuli were presented serially (see Figure 1), this explanation requires that proximity still facilitates noticing small differences between stimuli even when members of a pair are never seen simultaneously. Although further research is needed to test this assumption, it seems plausible in light of research showing that the spatial location of visually presented information is automatically indexed in memory and accessed during retrieval, even when the spatial information is task irrelevant (e.g., Richardson \& Spivey, 2000). 
APPENDIX A

Abstract Noun Pairs Used in Experiment 1

\begin{tabular}{lll}
\hline $\begin{array}{l}\text { advice-definition } \\
\text { betrayal-harm }\end{array}$ & $\begin{array}{l}\text { guess-affection } \\
\text { hesitation-thought }\end{array}$ & $\begin{array}{l}\text { moral-plan } \\
\text { motive-cause } \\
\text { boredom-loyalty }\end{array}$ \\
$\begin{array}{l}\text { hostility-mercy } \\
\text { chaos-prestige }\end{array}$ & opinion-philosophy \\
clue-paradox & idea-mystery & pact-exception \\
deduction-glory & ignorance-dread & pride-duty \\
delight-passion & impatience-insistence & sensation-concept \\
dignity-vanity & impetus-chance & sympathy-love \\
envy-heroism & integrity-equity & tendency-suspicion \\
excuse-wish & justice-grief & truth-theory \\
fact-illusion & magic-ability & value-gratitude \\
gratitude-prediction & memory-hope & vigor-obedience \\
\hline
\end{tabular}

\section{APPENDIX B}

Object Pairs Used in Experiment 3, From Snodgrass and Vanderwart (1980) Line Drawings

\begin{tabular}{lll}
\hline \multicolumn{1}{c}{ Clothing } & Kitchen Utensils & \multicolumn{1}{c}{ Tools } \\
\hline $\begin{array}{l}\text { crown-cap } \\
\text { dress-skirt }\end{array}$ & bottle-pitcher & axe-chisel \\
glove-sock & cup-glass & hammer-nail \\
fork-spoon & pliers-wrench \\
jacket-sweater & pan-bowl & scissors-saw \\
tie-bow & pot-kettle & screw-screwdriver \\
\multicolumn{1}{c}{ Furniture } & Musical Instruments & Vehicles \\
\hline bed-couch & accordion-piano & airplane-helicopter \\
chair-stool & drum-bell & bicycle-motorcycle \\
desk-dresser & flute-harp & bus-train \\
table-door & trumpet-horn & car-truck \\
vase-lamp & violin-guitar & wagon-sled \\
\hline
\end{tabular}

(Manuscript received July 10, 2007;

revision accepted for publication April 5, 2008.) 